\section{An Introduction to 3D Microscopy Techniques}

Megan MacNeil and Duncan McMillan

Carl Zeiss MicroImaging, Inc. Thornwood, NY.

MMacNeil@zeiss.com

\section{From Confocal Microscopy to Deconvolution}

In the earliest years of microscopy, it was well understood that visualization of the finest details was often limited by the intrusion' of light from out-of-focus planes. For many decades the only solution to this dilemma was to limit microscopic studies to very thin samples or to physically squash or slice thicker samples. More recently, biomedical research has driven the need for microscopes that can resolve very fine detail in 3 dimensions within intact, and often living, specimens. The use of fluorescence labeling further exacerbates the problem of out-of-focus light because signal is generated throughout the volume of the sample. The following article describes the most prevalent techniques for $3 \mathrm{D}$ imaging and important optical considerations for achieving highest image quality.

\section{Confocal}

The distinctive optical design feature of a confocal laser scanning microscope (LSM) compared to a conventional microscope is the confocal aperture (or pinhole) arranged in a plane conjugate to the intermediate image plane and the object plane of the microscope. The detector only detects light that passes through the pinhole - i.e. light that emanates from the object plane in focus. Because light from above and below the object plane is blocked, the confocal microscope can be understood as an inherently depth-discriminating - or sectioning - optical system.

By varying the pinhole diameter, the thickness of the optical section can be adapted to practical requirements - for example, the pinhole diameter can be increased to collect a thicker section and hence more light. As an added advantage, the pinhole suppresses stray light, which improves image contrast. Application advantages of the confocal approach are the possibility to acquire multiple channels simultaneously (depending on the number of detectors), the use of laser for photo manipulation (e.g. FRET) and the immediate access to the confocal image. Disadvantages include the high light energy in the scanned pixel, the costs for lasers and the relative complexity of the systems

\section{2-Photon}

2-photon (2P) microscopy is founded on the fundamental principle of $2 \mathrm{P}$ excitation that is, the absorption of two photons, at almost exactly the same time, with a fluorescent molecule. The combination of their energies causes the molecule to fluoresce as though it had been excited by a single photon of half the wavelength At typical microscope light intensities the probability of this occurring is extremely low, but by temporally compressing the light into short, but very intense, pulses the photon density in the focal volume is high enough to generate significant fluorescence. However, outside of the focal volume the photon density, and hence the generation of fluorescence, drops off dramatically. Therefore, while the $2 \mathrm{P}$ microscope is in many ways similar to a confocal microscope, the optical section formation itself is very different; in the confocal case fluorescence is generated throughout the vertical, or $\mathrm{Z}$, axis and the pinhole eliminates the unwanted light from above and below the object plane. In the $2 \mathrm{P}$ case, fluorescence is only generated in the object plane, thereby eliminating the need for a pinhole.

This critical difference means that $2 \mathrm{P}$ imaging is a more efficient technique - only the fluorophores in the object plane are excited, thus reducing the net photo-bleaching and photo-toxicity caused during a $3 \mathrm{D}$ acquisition. The other key benefit of $2 \mathrm{P}$ imaging is deeper penetration (100's of microns) due to the reduced scattering of the longer wavelength (typically $700-1000 \mathrm{~nm}$ ) light. $2 \mathrm{P}$ is the method of choice for imaging deep in opaque tissues, as necessary for intravital experiments.

\section{Structured illumination}

In addition to the different laser-based optical sectioning techniques, there are also several widefield methods. One such technique is structured illumination or "grid projection." The principle of structured illumination centers around a grid of parallel lines projected into the image plane - thus, a high-resolution objective images the grid lines in the same focal plane as the sample. A CCD sensor captures this data as well as the out-of-focus data that stems from above or below the focal plane. To calculate the optical section, three images are captured, each with the grid shifted by one-third. Within this set of images, the out-of-focus data appears nearly identical. It is subtracted out of the image, whereas the in-focus data is an addition of the subtracted images.

Using this technique, there are several factors that determine the optical section thickness, including: magnification and numerical aperture of the objective, wavelength, and the line pairs per millimeter of the grid. The application limitations are the rela-

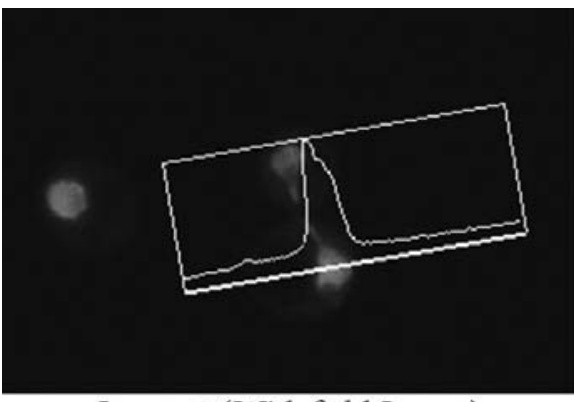

Image 1 (Widefield Image)

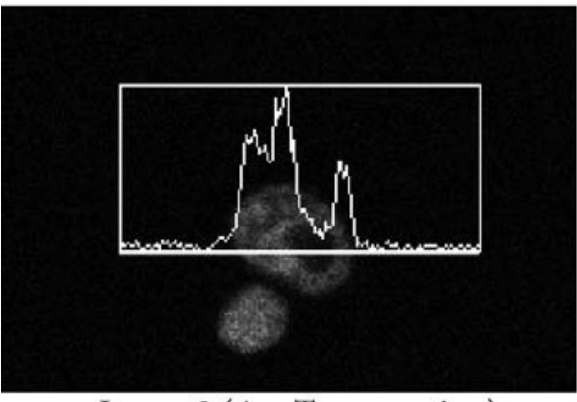

Image 3 (ApoTome section)

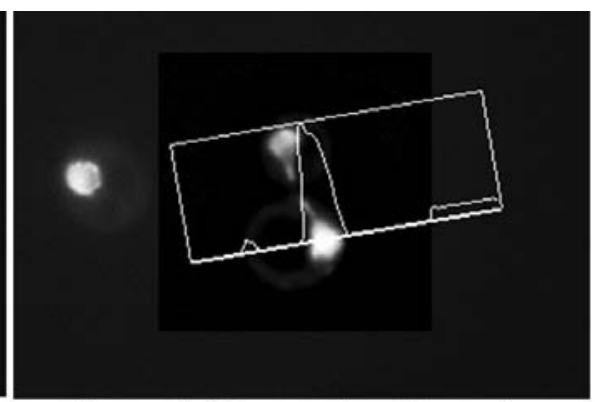

Image 2 (Deconvolved Image)

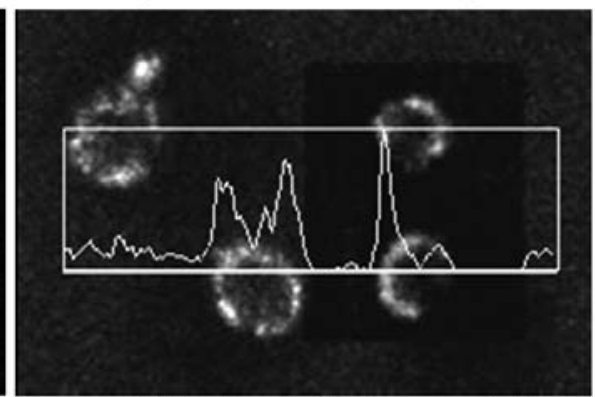

Image 4 (Confocalwith Deconvolution)
Caption: Images 1 to 4 show yeast cells with GFP transfected inner centromere proteins using conventional widefield (Image 1), Deconvolution in the Region of Interest (Image 2), Grating Imaging with the ApoTome (Image 3), and Confocal Imaging with Deconvolution in the Region of Imterest (Image 4). Images 1 to 4 were acquired with the Plan-Apo 63x/1.4 NA Objective. 


\section{POWER THROUEH SIMPLIGTY - AN IMAGING GOLUTION YOU COULD ONLY DAEAM ABOUT}

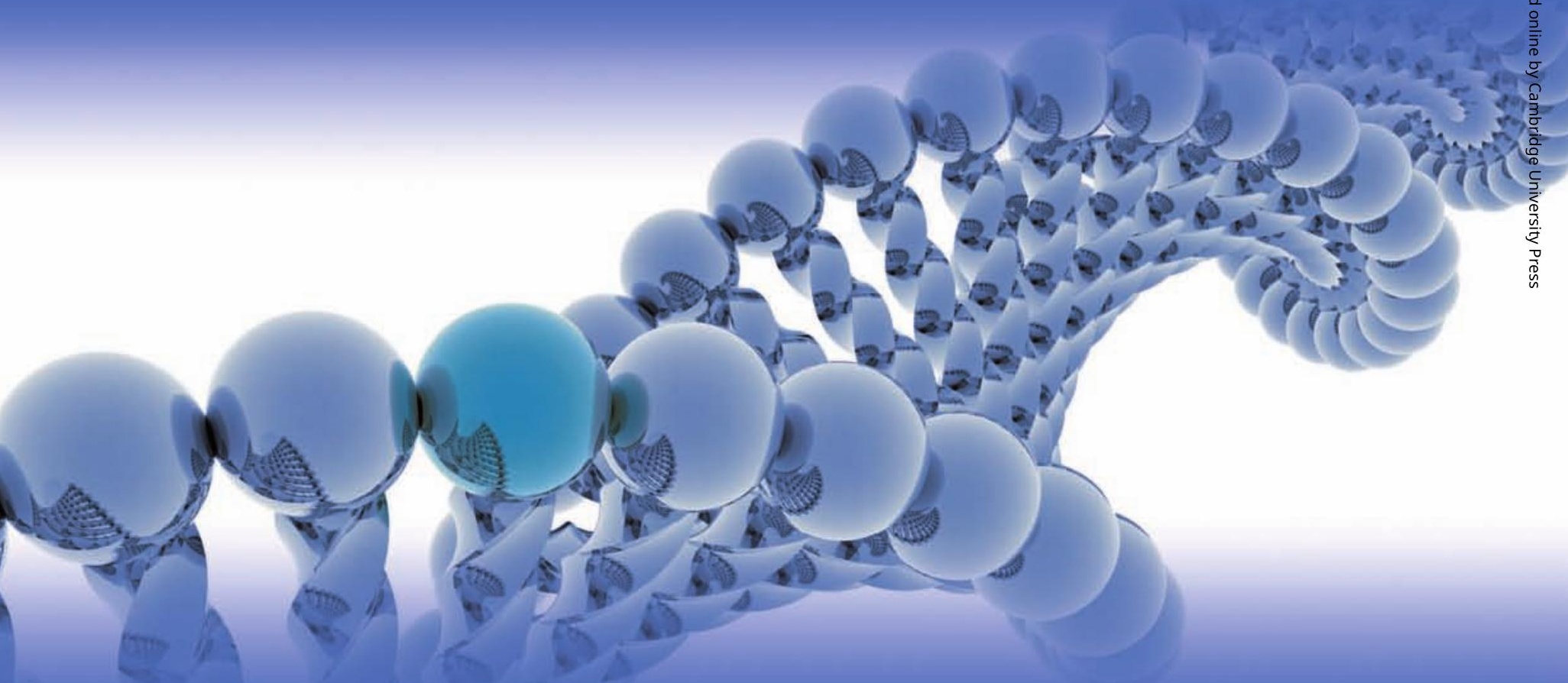

\section{ZEISS CENTRA® 100}

An efficient, fully digital, and robust multi-purpose $100 \mathrm{kV}$ TEM.

Designed for rapid biomedical applications.

CENTRA $^{\oplus} 100$ delivers outstanding image quality.
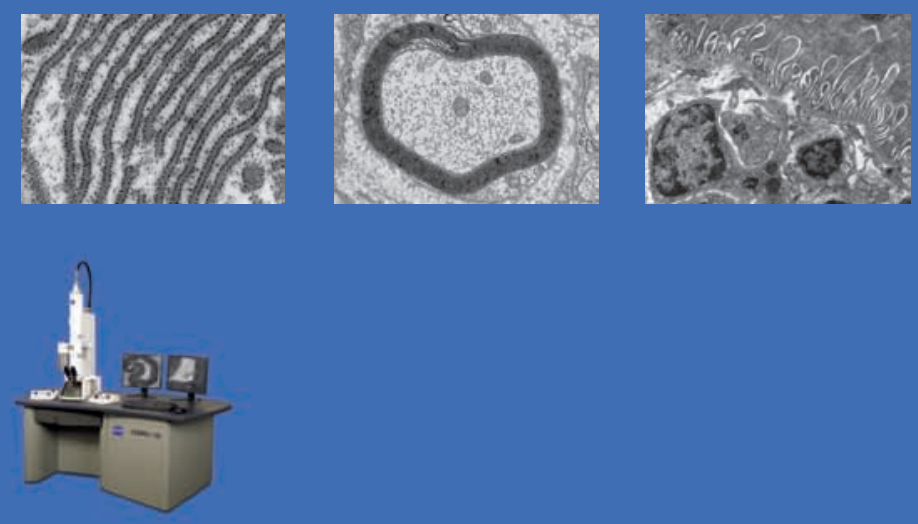
tive slow image acquisition and the need to acquire images sequentially (for fluorescence channels and grid positions). This limits the use for fast live cell imaging applications (>4fps), but allows an easy and convenient high resolution imaging of fixed or slow moving samples at very limited cost.

\section{Deconvolution}

A computational method to optical sectioning is deconvolution. Unlike the methods described above, deconvolution is purely mathematical. The basis of the calculations is the point-spread function, or the diffraction pattern of a point of light in three dimensions. Point spread functions vary from system

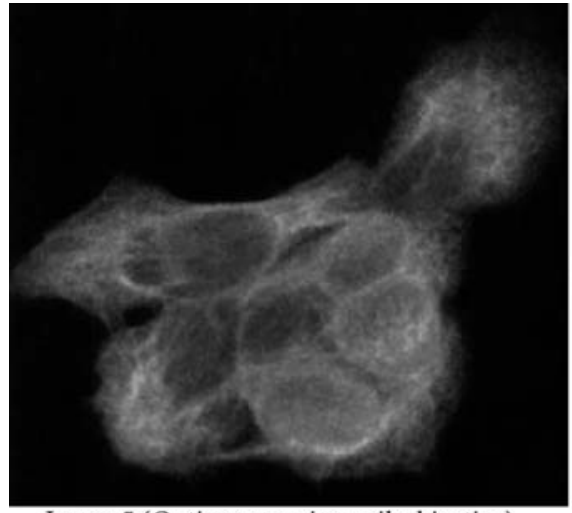

Image 5 (Optic comparison oil-objective)

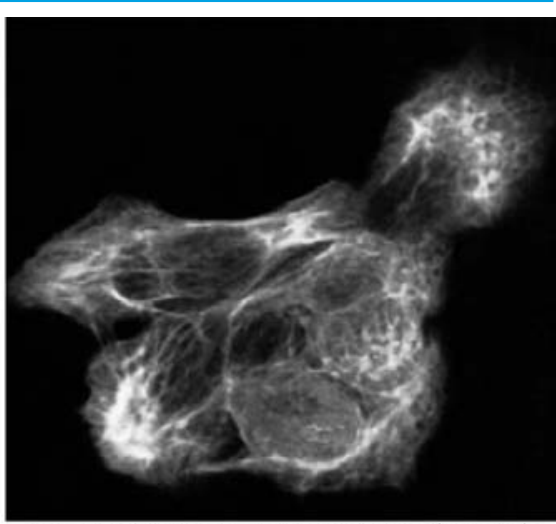

Image 6 (Optic comparison water-objective) to system, as the properties of the objective, wavelength of light, alignment of the microscope all play a role. Blur characterized by the point spread function is mathematically modeled as a convolution, and the convolution is applied to every point of light within the image. Deconvolution reverses this phenomenon and deblurs or reconstructs the data.

There are many different published deconvolution algorithms, but they segment into two groups: debluring or restorative. Debluring methods are $2 \mathrm{D}$ methods, where each $X Y$ plane is deconvolved individually, using image information only from that plane or the closest neighboring planes. Debluring calculations are much quicker calculations then their reconstructive counterparts. Restorative deconvolution, takes into account that blur is a $3 \mathrm{D}$ problem, and utilizes 3D data to subtract blur or reassign it to its original point of origin.

As an image processing approach, deconvolution does not immediately display a 'convolution free' image. But with increasing computer power this methods has again gained new application, especially for low light and fast imaging set ups. The strength of the restorative methods is to reassign the photons to the original source, gaining intensity and resolution.

\section{Important optical considerations for 3D applications}

Microscope objectives are generally designed to ensure best optical quality just beneath the cover slip. To reach higher magnifications, an immersion media is applied, mostly oil with the refractive index of the glass. Imaging three dimensional and thicker specimens normally results in a gradual loss of image quality with increasing penetration depth into the sample. The signal to noise ratio decreases and the image is of lower contrast.

Other effects include the reduction of resolution as well as an increasing error in calibration precision in the Z-Axis. This results in a reduced $Z$-extension of the cell, if the image is displayed in a rendering program, so e.g. the nucleus is flattened in the $Z$-projection. All of these effects are caused by scattering light in the sample and on the surface borders between embedding media, cover slip and immersion media and is called spherical aberration. It can be easily observed by comparing the shape of the airy disc of a small fluorescence particle above and below the object. Spherical aberration is a problem if the disks are not symmetrical. In order to reduce this effect and to optimize image quality as well as image true spatial locations of the fluorophores different strategies can be applied:

Optimize the optical light path for minimal refractive index mismatch. The refractive index of the immersion media should resemble that of the embedding media as closely as possible. Most common are water- or glycerin immersion objectives.

For a better adjustment, some of the objectives are equipped with a correction collar to precisely adjust the refractive index. Special live cell objectives combine high numerical apertures with Water or Glycerin Immersion (LCI Plan-Neofluar 63x/1.25 Imm. Corr.).

High aperture objectives are highly sensitive to differences in cover slip thickness. The use of closely tolerant cover slip thicknesses helps to avoid additional deteriorating effects in the images

Furthermore, imaging software can be used to correct for spherical aberration. The Deconvolution module in AxioVision offers the ability to correct for refractive Index mismatch, as well as the effects of increasing depth of the focal plane in the sample.

Please visit www.zeiss.com/3dimaging to find out additional information on applications, techniques, and 3D imaging solutions from Carl Zeiss. Please visit www.zeiss.com/objectives to for detailed information on microscope objectives from Carl Zeiss.

\section{SYMPOSIUM ANNOUNCEMENT Teaching Microscopy and Microanalysis Microscopy \& Microanalysis '08 August $3^{\text {rd }}-7^{\text {th }}$, Albuquerque, NM}

- How do we learn microscopy and microanalysis methods? - How do we effectively transfer our knowledge to those new to microscopy?

- How do we inspire students to enter and remain in the field?

- How do we keep up with advances in microscopy?

These are just a few of the questions that we hope to address in the symposium, "Teaching Microscopy and Microanalysis", to be held at M\&M 2008 in Albuquerque, New Mexico. We will explore issues facing those interested in teaching and learning light, electron, and analytical microscopies. Best practices in classroom teaching, technician training, short courses, remote learning, community outreach and vendor training are among the areas that will be discussed. Contributed papers are welcome.

Submission and registration information are available in the M\&M 2008 Call for Papers and on the Microscopy Society of America website, www.microscopy.org. Specific inquiries may be addressed to the organizers:

Charles Lyman, Lehigh University, charles.lyman@lehigh.edu

Elaine Schumacher, McCrone Associates, eschumacher@mccrone.com 


\section{Detectors from Oxford Instruments - Performance beyond the expected}

\section{Are you choosing the}

right EDS detector?

To ensure accurate and consistent results you can trust, you need the right detector for your application.

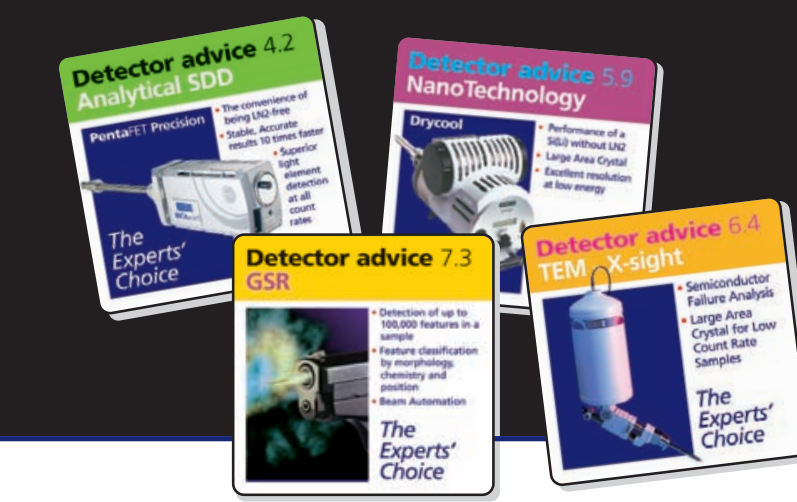

Email our specialists at nanoanalysis@oxinst.com to find out which detector you should choose, or visit our website at www.oxford-instruments.com/eds

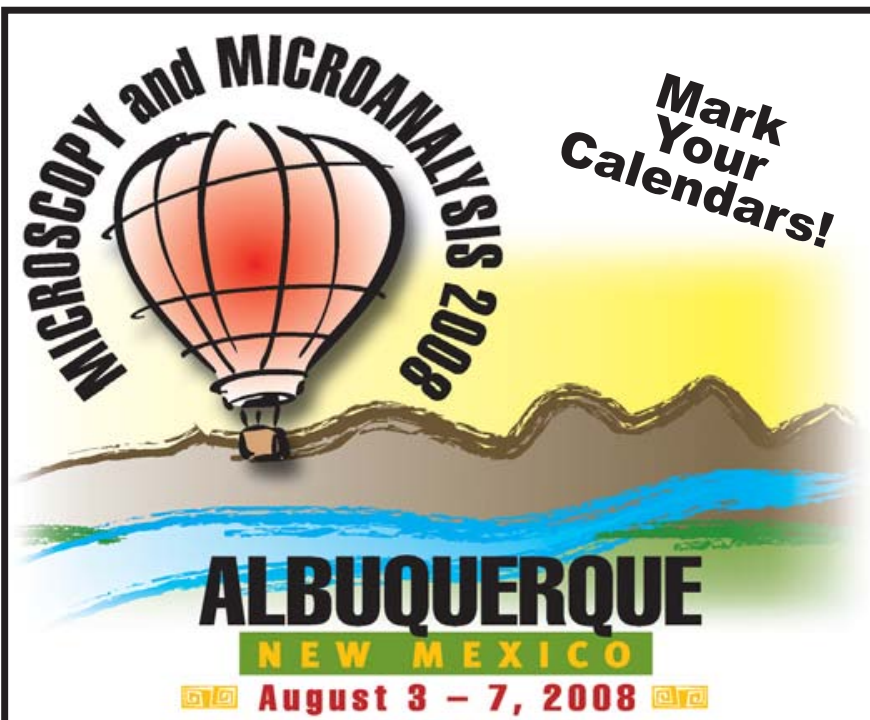

DID YOU RECEIVE TWO COPIES OF THIS ISSUE OF MICROSCOPY TODAY?

We have incorporated the USA attendance list from the Microscopy and Microanalysis-2007 meeting in Fort Lauderdale into the MT subscriber database. We have tried to eliminate duplicates, but we suspect that we were not $100 \%$ succesful. If you received two copies of MT, please notify microscopytoday@tampabay.rr.com to have the duplicate list entry removed. Thanks!

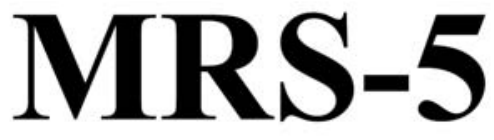

We are ISO-9000 certified and ISO-17025 accredited Microscopy Calibration Standard

Now you can calibrate from $1,000 X$ to $1,000,000 X !$

This is our fourth generation, traceable, magnification reference standard for all types (SEM, FESEM, Optical, STM, AFM, etc.) of microscopy. The MRS-5 has multiple $\mathrm{X}$ and $\mathrm{Y}$ pitch patterns ranging from $80 \mathrm{~nm}( \pm 1 \mathrm{~nm})$ to $2 \mu \mathrm{m}$ and $3 \mathrm{bar}$ targets from $80 \mathrm{~nm}$ to $3 \mu \mathrm{m}$. There is also a STM test pattern.

Free web resource guide!
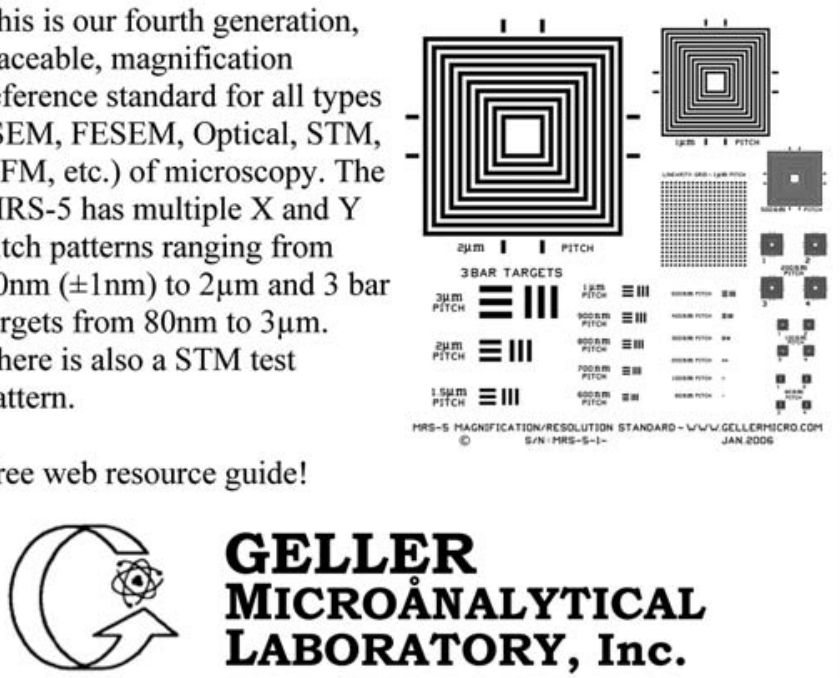

426e Boston St., Topsfield, Ma 01983 www.gellermicro.com 